\title{
True identity of control Staphylococcus aureus strains and their performance in the tube coagulase test
}

\author{
M. H. WILCOX*‡, C. WALKER*, T. G. WINSTANLEY ${ }^{\dagger}$ and D. I. LIMB ${ }^{\dagger}$ \\ * Clinical Microbiology and Public Health Laboratory, Level 6, Addenbrooke's Hospital, Cambridge CB2 $20 W$ \\ and ${ }^{\dagger}$ Bacteriology Department, Royal Hallamshire Hospital, Sheffield S10 2JF
}

\begin{abstract}
One hundred laboratories were asked to submit their control Staphylococcus aureus strains to determine the true identity of strains presumed to be $S$. aureus NCTC 6571, and also to evaluate the performance of those strains being used as controls in the tube coagulase test (TCT). Of the 60 who replied, 55 laboratories sent at least one strain labelled as $S$. aureus NCTC 6571 (total of 64 strains). Of these, $84 \%$ were identified as $S$. aureus, and were indistinguishable from a fresh type strain by a combination of phenotypic methods including biotyping, antibiotic susceptibility testing and phage typing. Six-to-ten strains (9-16\%), depending on the degree of stringency, were not identifiable as $S$. aureus NCTC 6571 . The time since last retrieval from storage ranged from daily to $\geqslant 3$ years, but there was no correlation between this duration and the likelihood of differing from $S$. aureus NCTC 6571. Forty-seven laboratories submitted 51 strains used as controls in the TCT; these included 31 strains labelled as $S$. aureus NCTC 6571, eight wild strains, three other NCTC strains and nine strains of uncertain origin. Generally, the $S$. aureus NCTC 6571 strains produced weaker clots than the remainder. None of the $S$. aureus NCTC 6571 strains was found to be inoculum dependent but four of the other control strains were. The study demonstrates that some laboratories must improve procedures for ensuring that control $S$. aureus strains retain their true identity, particularly by avoiding repeated subcultures. Laboratories are divided in their use of strong or weak (S. aureus NCTC 6571) positive controls for the TCT. S. aureus NCTC 6571 is a more stringent control for the TCT than other control strains presently being used and is, therefore, to be preferred.
\end{abstract}

\section{Introduction}

Although there are a few reports of Staphylococcus aureus strains that give negative results in the tube coagulase test (TCT) [1,2], this method is still generally considered as the gold standard for distinguishing between coagulase-positive and coagulasenegative straphylococci in the clinical microbiology laboratory. Several factors have been identified as potential causes of false-negative and false-positive results in the TCT, including inoculum size, temperature, humidity, $\mathrm{CO}_{2}$ concentration, variations in the sensitivity of rabbit plasma and production of proteases or pseudocoagulase [3-6]. However, the choice of

Received 17 Aug. 1995; revised version accepted 26 Oct. 1995. ${ }^{\ddagger}$ Present address and address for correspondence: Department of Microbiology, University of Leeds, Leeds LS2 9JT. control strains may also be important for the correct interpretation of results. For example, use of a positive control strain that produces a weak rather than a strong clot is a more stringent test of the method, but is likely to lead to more tests needing to be repeated, because of false-negative results with the positive control. Repeated subculture of control strains is, of course, a risk factor for the acquisition of strains with altered phenotype, and drift or shift in the expression of free coagulase by control strains may have important consequences. Recently, a change was observed in the performance of the $S$. aureus control (a wild strain) in the TCT in this laboratory, with either no clot or only weak clots occurring.

Other laboratories were surveyed and it was found that $S$. aureus NCTC 6571 was the most commonly used control strain in the TCT. Therefore, the true identities 
of control strains presumed to be $S$. aureus NCTC 6571 (the 'Oxford straphylococcus') were investigated in this study. The performance of strains which were being used as controls in the TCT by clinical microbiology laboratories in the UK was also compared.

\section{Materials and methods}

\section{Sources of strains}

One hundred randomly selected, clinical microbiology laboratories in the UK were asked to send in their control $S$. aureus strains, to indicate for which tests they were being used as controls, and to document the dates when these strains were last subcultured and retrieved from storage. Strains submitted were stored at $4^{\circ} \mathrm{C}$ on their original nutrient agar slopes, and were only subcultured, on horse blood $5 \%$ agar plates, the day before testing. A fresh $S$. aureus NCTC 6571 strain was obtained from the National Collection of Type Cultures (Central Public Health Laboratory, 61 Colindale Avenue, London).

\section{Strain identification}

The following methods were used to determine the identity of strains submitted.

Tube coagulase test. The standard method used in this laboratory was used for the TCT, which is a slightly modified version of one of the four alternatives given in Cowan and Steel's Manual for the Identification of Medical Bacteria [7, 8]. Two colonies from an overnight culture were emulsified in $1 \mathrm{ml}$ of a mixture of fresh EDTA rabbit plasma (Advanced Protein Products, Brierley Hill, W. Midlands $10 \% \mathrm{v} / \mathrm{v}$ ), phosphate buffered saline, $\mathrm{pH} 7.2,75 \% \mathrm{v} / \mathrm{v}$ and brain heart infusion broth (Difco $15 \% \mathrm{v} / \mathrm{v}$ ), in a $10 \times 1.2$-cm glass tube. Clot formation was recorded after incubation for $4 \mathrm{~h}$ in a water bath at $37^{\circ} \mathrm{C}$, and again at $24 \mathrm{~h}$ after incubation in air at room temperature $\left(22^{\circ} \mathrm{C}\right)$. Clot formation was graded as,,$++++l-$, or - , corresponding to the clot sticking to the side of tube when this was tilted horizontally, the clot not sticking to the tube, weak clot formation, or no clot, respectively. The following inocula were compared in the TCT: one and two colonies from an overnight culture, and a 1 in 10 dilution of one colony (prepared by transferring $0.1 \mathrm{ml}$ from a tube containing a single emulsified colony to a second tube containing $0.9 \mathrm{ml}$ of plasma/saline/broth).

Antimicrobial susceptibility testing. Susceptibility to 19 antibiotics was determined by agar incorporation and multipoint inoculation.

Phage typing. Lysis by the international set of staphylococcal bacteriophages (obtained from CPHL, Colindale Avenue, London) was determined at routine test dose.
Biotyping. Biotyping with API Staph test strips (BioMérieux, Basingstoke) was performed according to the manufacturer's instructions. Additional biotyping, including DNAase production, was by agar incorporation and multipoint inoculation.

\section{Results \\ Identification of 'NCTC 6571' strains}

Replies were received from 60 laboratories with a total of 170 control strains labelled as $S$. aureus. Of these 170 strains, 64 from 55 laboratories were claimed to be S. aureus NCTC 6571 . Fifty-two of these 64 strains were confirmed as NCTC 6571 according to API Staph profile number $(6736153)$, phage type $(52 \mathrm{~A}, 79)$ and supplementary biochemical tests. Of the remaining 12 strains, six were distinguishable from the type strain by at least two different test results (one strain was urease negative and therefore had a different API Staph profile number, 6736151). Four strains macroscopically looked unlike the type strain, consistently gave one equivocal result in the API Staph strip and differed by one phage reaction. The remaining two strains each differed by only one phage reaction, and therefore were not considered to be different form the type strain $S$. aureus NCTC 6571. Hence, depending on the degree of stringency applied, between $9 \%$ ( 6 of 64$)$ and $16 \%$ (10 of 64) of the submitted strains which were claimed to be $S$. aureus NCTC 6571 could not be confirmed as such. No differences were seen between the antibiograms of strains submitted as $S$. aureus NCTC 6571 .

Each of the 10 strains labelled as $S$. aureus NCTC 6571 , but which could not be identified as such, were being used as controls in antibiotic susceptibility tests in their laboratories of origin. Two strains were also being used as controls in the TCT, and these were confirmed as TCT-positive. Of the strains used as controls in antibiotic susceptibility tests, one gave weak and two a negative result in the TCT. There was no correlation between the duration since strains had last been recovered from storage and the likelihood of differing from $S$. aureus NCTC 6571 (Table 1).

\section{Comparison of strains in the tube coagulase test}

Forty-seven laboratories submitted 51 strains used as controls in the TCT (four laboratories sent more than one positive control strain). These comprised $31 \mathrm{~S}$. aureus NCTC 6571, eight wild strains, two $S$. aureus NCTC 8532 , one $S$. aureus NCTC 8178 and nine strains of unknown origin. Comparison of these control strains in the TCT and the results from different inocula are shown in Table 2. Generally, control strains confirmed as $S$. aureus NCTC 6571 produced weaker clots than did other $S$. aureus strains. Clot formation by the $S$. aureus NCTC 6571 strains was not inoculum dependent, whereas four of the other control strains (all wild-type strains) produced weaker or no clots when 
Table 1. Effect of time elapsing between data of recovery from storage and correctness of identity as $S$. aureus NCTC 6571

\begin{tabular}{|c|c|c|c|}
\hline $\begin{array}{l}\text { Time since last } \\
\text { recovered from storage }\end{array}$ & $\begin{array}{l}\text { Number of strains confirmed } \\
\text { as } S . \text { aureus NCTC } 6571\end{array}$ & $\begin{array}{l}\text { Number of strains differing } \\
\text { from } S \text {. aureus NCTC } 6571\end{array}$ & $\begin{array}{l}\text { Total numbers } \\
\text { of strains }\end{array}$ \\
\hline$<1$ month & 23 & 3 & 26 \\
\hline $1-6$ months & 10 & 2 & 12 \\
\hline 6 months- 1 year & 3 & 1 & 4 \\
\hline$>1$ year & 3 & 2 & 5 \\
\hline No data & 6 & 1 & 7 \\
\hline Not known & 9 & 1 & 10 \\
\hline Total & 54 & 10 & 64 \\
\hline
\end{tabular}

smaller inocula were used. S. aureus NCTC 8532 and $S$. aureus NCTC 8178 produced stronger and weaker clots than $S$. aureus NCTC 6571 , respectively. Clot formation did not increase between $4 \mathrm{~h}$ and $24 \mathrm{~h}$ for any of the strains tested.

\section{Discussion}

Phenotypic methods were specifically used to attempt to discriminate between the strains submitted as $S$. aureus NCTC 6571. Although genotypic methods are more discriminatory, the strains were being used as controls in phenotypic tests, and we wished to detect changes in behaviour in the latter. The study demonstrated that $9-16 \%$ of the strains submitted as $S$. aureus NCTC 6571 were phenotypically different from the type strain. None of these, which were being used in the laboratory of origin as controls in the TCT, had altered behaviour in this test in our hands. Similarly, no changes were observed in the antibiograms of the strains submitted as $S$. aureus NCTC 6571, despite other variations in phenotype. This probably reflects the low MICs of antibiotics for this strain. This explains why those laboratories that sent strains labelled as $S$. aureus NCTC 6571 , but which could not be confirmed as such, failed to detect alterations in phenotypic behaviour, as the majority were using these strains as controls in antibiotic susceptibility testing. Although the response rate in the survey was only $60 \%$, this is fairly typical of such studies, and we have no reason to believe that our findings have been biased.

It could be argued that the finding of strains being wrongly assumed to be $S$. aureus NCTC 6571 is of little consequence, because on the whole the strains would still give correct control results. However, $S$. aureus NCTC 6571 may be used to control a wide variety of methods, only a few of which were addressed in the present study, and it is assumed that the phenotype of a control strain is stable. We were unable to demonstrate a significant association between the time elapsed since $S$. aureus NCTC 6571 strains had last been recovered from storage and the likelihood of these differing from the type strain. Nevertheless, it was not surprising to observe a trend towards an increased likelihood of misidentification of this extensively used control strain in those laboratories that last recovered the strain more than, as opposed to less than, 1 month previously ( $24 \%$ versus $12 \%, p>0.1)$. It is unacceptable that in many laboratories this period was in excess of 1 month, and indeed in $9(14 \%)$ of 64 instances it was $>6$ months. Some laboratories are performing excessive subcultures without recourse to stored control strains. Good laboratory practice with control strains should include a system whereby only a limited number of subcultures is permitted, at which point fresh strains are retrieved from cold storage. Beads for storing strains frozen are particularly useful in this context. It is clear that some laboratories should improve their procedures for ensuring that control $S$. aureus strains retain their true identity.

S. aureus NCTC 6571 strains produced weaker clots than other control strains. The inoculum effect was confirmed with four control strains in the TCT, as described in an earlier study [6] of clinical isolates,

Table 2. Results of TCT* $^{*}$ with $S$. aureus NCTC 6571 and other $S$. aureus control strains

\begin{tabular}{|c|c|c|c|c|c|c|c|c|}
\hline \multirow[b]{2}{*}{ Inoculum size } & \multicolumn{4}{|c|}{$\begin{array}{l}\text { 'S. aureus NCTC } 6571 \text { '† } \\
(\mathrm{n}=31)\end{array}$} & \multicolumn{4}{|c|}{$\begin{array}{l}\text { Other } S . \text { aureus strains } \\
\qquad(\mathrm{n}=19)^{\ddagger}\end{array}$} \\
\hline & ++ & + & $+1-$ & - & ++ & + & $+1-$ & - \\
\hline 2 colonies & 0 & 31 & 0 & 0 & 15 & 3 & 1 & 0 \\
\hline 1 colony & 0 & $10^{\S}$ & 0 & 0 & 15 & 0 & 1 & 3 \\
\hline 0.1 colony & 0 & $10^{\S}$ & 0 & 0 & 15 & 0 & 1 & 3 \\
\hline
\end{tabular}


whereby some $S$. aureus strains only give a positive result if two colonies, rather than one, are used. However, none of the $S$. aureus NCTC 6571 strains exhibited this phenomenon. It has been shown that $S$. aureus does not multiply in rabbit plasma [6], although this may only be true if incubation occurs in air without additional $\mathrm{CO}_{2}$, in which case the $\mathrm{pH}$ of plasma would be expected to increase above physiological levels by $1-2 \mathrm{pH}$ units [9]. Interestingly, some methods for the TCT nevertheless recommend that only a single colony is used $[7,8,10]$, presumably to ensure that only one strain is sampled in the case of mixed bacterial populations. Of those laboratories who replied to the present survey, $62 \%$ use $S$. aureus NCTC 6571 as a positive control in the TCT. The great majority of the remainder use control strains which, in our hands, gave stronger clots. We conclude that the use of $S$. aureus NCTC 6571 is a more stringent control for the TCT and is therefore to be preferred. Laboratories that continue to use control strains that give stronger clots risk false-negative results. Repeated subculturing of control strains should be avoided and instead these should be recovered frequently from stored stocks.

We thank the laboratories who participated in our survey for making this study possible.

\section{References}

1. Barrett SP. Rate of isolation of Staphylococcus aureus strains possessing coagulase of clumping factor. Eur J Clin Microbiol 1983; 2: 475-476.

2. Vandenesch F, Bes M, Lebeau C, Greenland T, Brun Y, Etienñe J. Coagulase-negative Staphylococcus aureus. Lancet 1993; 342: 995-996.

3. Thomas MEM. Studies on a $\mathrm{CO}_{2}$-dependent Staphylococcus. $J$ Clin Pathol 1955; 8: 288-291.

4. Landau W, Kaplan RL. Room temperature coagulase production by Staphylococcus aureus. Clin Microbiol Newsletter 1980; 2: 10.

5. Wegrynowicz Z, Heczko PB, Jeljaszewicz J, Neugebauer M, Pulverer G. Pseudocoagulase activity of staphylococci. J Clin Microbiol 1979; 9: 15-19.

6. Selepak ST, Witebsky FG. Inoculum size and lot-to-lot variation as significant variables in the tube coagulase test for Staphylococcus aureus. J Clin Microbiol 1985; 22: 835837.

7. Barrow GI, Feltham RKA (eds). Cowan and Steel's Manual for the identification of medical bacteria, 3rd edn. Cambridge, Cambridge University Press. 1993: 226.

8. Subcommittee on taxonomy of staphylococci and micrococci. Recommendations. Int Bull Bact Nomencl Taxon 1965; 15: 109-110.

9. Wilcox MH, Schumacher-Perdreau F. Lack of evidence for increased adherent growth in broth or human serum of clinically significant coagulase-negative staphylococci. $J$ Hosp Infect 1994; 26: 239-250.

10. Kloos WE, Bannerman TL. Staphylococcus and Micrococcus. In: Murray PR, Baron EJ, Pfaller MA, Tenover FC, Yolken RH (eds) Manual of clinical microbiology, 6th edn. Washington, American Society for Microbiology. 1995: 288-289. 